\title{
Vessel bend-based cup segmentation in retinal images
}

\author{
Gopal Datt Joshi, Jayanthi Sivaswamy \\ CVIT, IIIT Hyderabad, India \\ gopal@research, jsivaswamy@iiit.ac.in
}

\author{
Kundun Karan, Prashanth R., S. R. Krishnadas \\ Aravind Eye Hospital, Madurai, India \\ krishnadas@aravind.org
}

\begin{abstract}
In this paper, we present a method for cup boundary detection from monocular colour fundus image to help quantify cup changes. The method is based on anatomical evidence such as vessel bends at cup boundary, considered relevant by glaucoma experts. Vessels are modeled and detected in a curvature space to better handle inter-image variations. Bends in a vessel are robustly detected using a region of support concept, which automatically selects the right scale for analysis. A reliable subset called $r$-bends is derived using a multi-stage strategy and a local spline fitting is used to obtain the desired cup boundary. The method has been successfully tested on 133 images comprising 32 normal and 101 glaucomatous images against three glaucoma experts. The proposed method shows high sensitivity in cup to disk ratio-based glaucoma detection and local assessment of the detected cup boundary shows good consensus with the expert markings.
\end{abstract}

\section{Introduction}

Early detection and treatment of retinal diseases are crucial to avoid preventable vision loss. Glaucoma is one of the most common causes of the blindness and about 79 million in the world are likely to be afflicted with glaucoma by the year 2020. It leads to irreversible vision loss due to significant loss of optic nerve fibers.

Retinal nerve fibers converge to the optic disk (OD) and form a cup-shaped region known as the cup. Enlargement of this cup with respect to OD is an important indicator of glaucoma progression and hence ophthalmologists manually examine the OD and cup for evaluation. An automatic assessment of cup region from colour fundus image (CFI) could reduce the workload of experts and aid objective detection of glaucoma.

A CFI is a projection of retinal structures on $2 \mathrm{D}$ color plane where OD appears as a bright circular or elliptic region partially occluded by blood vessels as shown in fig 1(a). Given the loss of depth information in a CFI, glaucoma experts use a change in vessel morphology
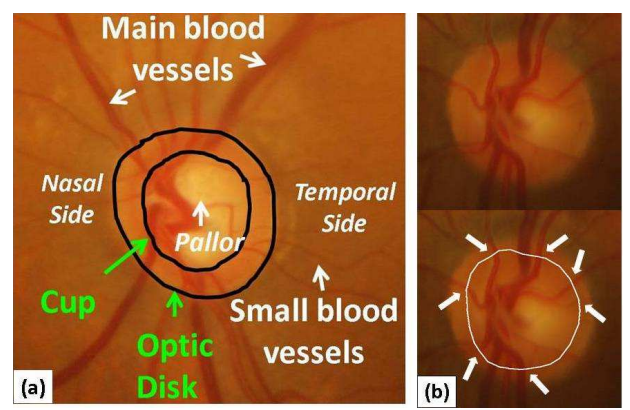

Fig. 1: a) OD-centric CFI b) Cup boundary through r-bends.

as a reliable visual clue for determining cup boundary. Thus cup segmentation is a time-consuming and challenging task. Figure 1(b) shows cup boundary marked a glaucoma expert using vessel bends (highlighted by arrows) information.

Much of existing work has mainly focused on OD segmentation with only few attempts at cup segmentation. Cup boundary detection has been proposed using 3-D depth information [5]. Since 3-D images are not easily available, Liu et. al. [3] proposed a cup boundary estimation method using monocular CFI. In this approach, a potential set of pixels belonging to cup region is first derived based on the reference colour obtained from a manually selected point. Next, an ellipse is fit to this set of pixels to estimate the cup boundary. A variant of this method obtains the cup pixels via thresholding of the green colour plane [3]. Cup boundary obtained via ellipse fitting yields only coarse cup boundary. Furthermore, fixed thresholding is also not adequate to handle large inter-image intensity variations that arise due to complex imaging and physiological difference across patients.

In order to address these problems, additional information such as small vessel bends ('kinks') which anatomically mark the cup boundary have been used in [4]. Here, image patches are extracted around a estimated cup boundary obtained in [3] and vessel pixels are identified using edge and wavelet transform information. Next, vessel bends, characterized by points of direction change in the vessel pixels are found and used 


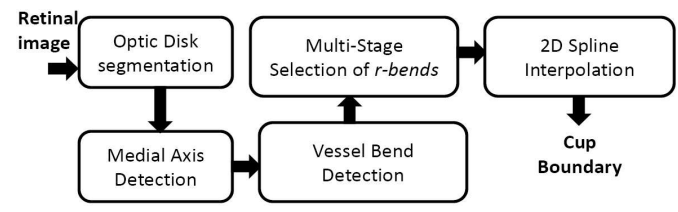

Fig. 2: The proposed method

to obtain the cup boundary. This method is highly dependent on the preliminary cup boundary obtained from [3]. Furthermore, the statistical rule for selecting vessel pixels is very sensitive to the inter-image variations.

Both appearance and anatomical knowledge are used by the glaucoma expert to determine cup boundary in different cup regions. Hence, we propose a method that integrates both information under a common framework. The cup is modeled as a region enclosing pallor region (shown in fig. 1(a)) and defined by a boundary passing through a sparse set of vessel bends. In the next section, we will explain proposed method in detail.

\section{Proposed Method}

Our objective is to segment the cup region by using anatomical evidences considered relevant by the experts. As seen in fig. 1(b) and cyan points in fig. 3(b), vessel bends can occur at many places within the OD region. However, only a subset of these points define the cup boundary. We refer to this as relevant vessel bend or $r$-bend. The first problem at hand is to find this subset. We use multiple source of information for this purpose: the pallor region which spatially defines the inner limit of $r$-bend, bending angle and location in the OD region. A second problem is that the anatomy of the OD region is such that the r-bends are non-uniformly distributed across a cup boundary with more points on the top and bottom; they are mostly absent in the nasal side and very few in number in the temporal side. We propose a local interpolating spline to naturally approximate the cup boundary in regions where $r$-bends are absent. Figure 2 shows an overview of the proposed method. The energy minimization based deformable models are not adequate due to absence of certain edge or region based information associated with the cup region to derive an energy functional.

Optic Disk Segmentation: The OD region is localised from red channel of the CFI and morphologicalbased pre-processing step is performed to reduce the effect of vessels within OD. We use the region-based active contour approach in [1] for segmenting the OD. The contour $C(s):[0,1] \rightarrow \mathbb{R}^{2}$, which is a piecewise parameterized $C^{1}$ curve, is evolved using an energy functional defined as

$$
\begin{aligned}
F\left(c^{+}, c^{-}, C\right)= & \mu \cdot \text { Length }(C) \\
& +\lambda^{+} \int_{\text {inside }(C)}\left|I_{0}(x, y)-c^{+}\right|^{2} d x d y \\
& +\lambda^{-} \int_{\text {outside }(C)}\left|I_{0}(x, y)-c^{-}\right|^{2} d x d y
\end{aligned}
$$

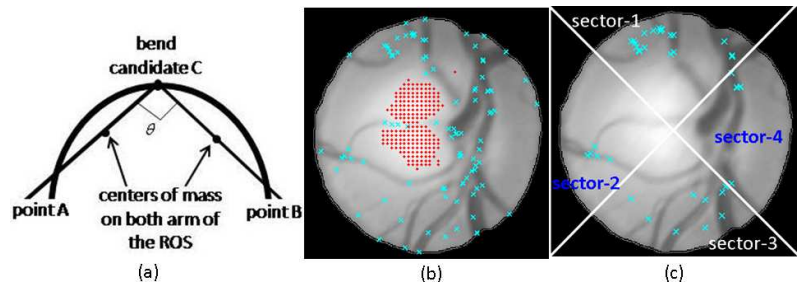

Fig. 3: a) Angle of a vessel bend, b) uniform pallor samples(red), bend points(cyan) and c) fitted circle(red) and potential r-bends.

where $c^{+}$and $c^{-}$are unknown constants representing the average value of $I_{0}$ inside and outside the curve, respectively. The parameters $\mu \geq 0$ and $\lambda^{+}, \lambda^{-} \geq 0$; are weights for the regularizing and the fitting terms, respectively. For curve evolution, the level set formulation is used where the motion is governed by mean curvature [1]. For further processing steps, we only consider green colour plane of segmented OD region.

Medial axis detection: The OD region has both thick and thin vessels and due to large inter-image variation, detecting both kinds is difficult. Hence, we embed the image in a 3D space and formulate the blood vessel detection as a problem of trench detection in the intensity surface. The selection of this space gives robustness to the image variations and detection is solely driven by trench shape and directional continuity associated with a vessel structure. Trenches are regions characterized by high curvature, oriented in a particular direction.

The curvature is computed using surface tangent derivative [2] defined as: $\Upsilon(x)=\frac{d^{2} y / d x^{2}}{1+(d y / d x)^{2}}$. For each point, $\Upsilon$ is computed in 4 different directions. The maximum value of the responses $\Upsilon_{\max }$ and corresponding orientation $\alpha$ (perpendicular to the vessel direction) are retained and further assessed to obtain trench points. A point is declared as a trench if value of $\Upsilon_{\max }$ is greater than both threshold value $t$ and the values of neighboring pixels in $\alpha$ direction.

For the robust detection of low contrast vessels, we employ a two-phase thresholding scheme in which first, a high value of $t$ is applied to get high contrast vessel points (set-1). Then, low value of $t$ is applied to get a new set of low contrast vessel points (set-2). Points in set- 2 which are found connected to the set- 1 are included in the final set along with set-1. This strategy helps in successfully extracting low contrast vessels while rejecting noise. The final trench points give a medial axis based representation of vessel structure which is more precise in quantifying vessel bends compared to edge-based representation. The next task is to extract vessel bends from this representation.

Vessel Bend detection: The amount of bending in vessels varies according to the caliber of vessel. Thin vessels show significant bending compared to a thick 
vessel. This is due to the fact that thick vessels are more rigid. The selection of appropriate scale for detecting both types of bend is crucial because bend in a thick vessel is apparent only at a larger scale compared to a bend in thin vessel. We employ a dynamic region of support (ROS) based scheme to find the appropriate scale to analyse a candidate point.

First, we extract vessel segments terminated by end and/or junction points. For each segment, we compute 1D shape (curvature) profile and locate the local maxima. These local maxima constitute a candidate set of bends $b$. A ROS for any $b_{i}$ is defined as a segment of vessel around $b_{i}$ and bound on either side by the nearest curvature minimum. Choosing the bounds to be based on curvature minima automatically ensures the size of the ROS to be large for thick vessels and small for thin vessels. The angle of bend $\theta$ is then computed as the angle between the lines joining a bend point and the centers of mass on both sides of the ROS. The center of mass of an arm is defined by the mean position of pixels on the arm (illustrated in fig. 3(a)). Since only vessels bending into the cup are of interest, bends above $\theta=170^{\circ}$ are eliminated from the candidate set. The detected vessel bends in a sample image are highlighted in fig. 3(b) with cyan markers.

Multi-stage selection of r-bends The task of identifying the $r$-bends from $b_{i}$ is performed in two stages, to reduce the required analysis, by utilizing anatomical knowledge associated with $r$-bends. In the first stage, a coarse selection is done based on a bend's proximity to the pallor region. In the second stage, the spatial position and bending information are used to identify the set of $r$-bends.

First stage: Let $p:\left(x_{p}, y_{p}\right)$ be a set of points within the pallor region. These were found by retaining the top $25 \%$ of the bright pixels within the OD. Next, let $b:\left(x_{b}, y_{b}\right)$ be the locations of the bends $b_{i}$. The region containing potential $r$-bends is localised by finding a best-fit circle (in least-square sense) to the set of points $(x, y)=\{p, b\}$. Let the circle have center $\left(x_{c}, y_{c}\right)$ and radius $R$. The minimisation of error function $S=\sum_{i}\left(\left(x_{i}-\bar{x}\right)-x_{c}\right)^{2}+\left(\left(y_{i}-\bar{y}\right)-y_{c}\right)^{2}-R^{2}$ gives the unknown parameters $x_{c}, y_{c}$ and $R$, where, $\bar{x}=\frac{1}{n} \sum_{i} x_{i}, \bar{y}=\frac{1}{n} \sum_{i} y_{i}$ and $n$ number of points. The bends which lie in the vicinity of this circle (inside and outside) are passed to the next stage. Figure 3(c) shows sample candidate $r$-bends obtained in this stage.

Second stage: Each candidate bend is analysed in terms of its sector-wise location (as in fig. 3(c)) and its parent vessel orientation. This analysis is based on anatomical knowledge that bends formed by vertical vessels in sec-1\&3 and horizontal vessels in sec- $2 \& 4$ are the probable $r$-bends. The final refined set of $r$-bends

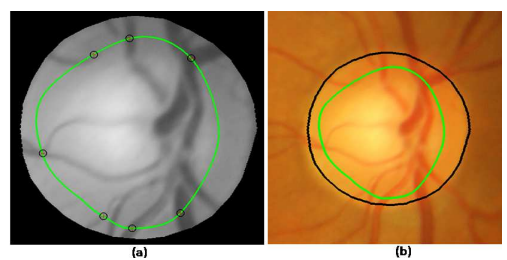

Fig. 4: a) Estimated cup boundary, b) final OD and cup boundary.

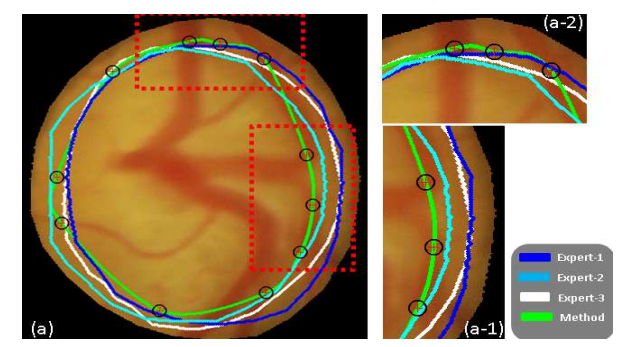

Fig. 5: Detected cup boundary.

is found as follows: A sector is radially analysed with a step size of $20^{\circ}$ and in each step, only bends formed by vessels with the 'correct' orientation are retained. If multiple bends remain, then the bend with smaller value of theta $\theta$ is selected as thin, rather than thick, vessel bends are more reliable indicators for the cup boundary. These usually occur in the diagonal region between two sectors.

2D spline interpolation Typically, $r$-bends are sparse and not uniformly distributed across the sectors. In their absence, experts use their clinical knowledge (experience of direct 3D cup examination) to approximate a cup boundary. Hence, it is difficult to get the cup boundary in the regions with no $r$-bends. We choose a local cubic cardinal spline, which is a generalisation of Catmull-Rom spline, with a shape parameter $t$. The parameter $t$ helps control the bending behaviour and thus the shape according to the sector. The value of $t$ is kept high in sectors $2 \& 4$ as they usually have low vessel density ( $r$-bends) compared to sector $1 \& 3$. A closed-form 2D spline curve is obtained by considering, sequentially, a subset of $r$-bends. Figure 4(a) shows the interpolated cup boundary passing through the $r$-bends and Fig. 4(b) shows final obtained boundaries for a sample OD region.

\section{Experimentation Results}

The proposed method was evaluated on a dataset of retinal images collected from an ongoing pilot study in collaboration with a local eye hospital. The dataset has 32 normal and 101 glaucomatous (total of 133) images. All images were taken under a fixed protocol with 30degree field of view, centered on the OD. For each image, ground truth was collected from three glaucoma experts, referred to as E1, E2 and E3 with experience of 3,5 and 20 years, respectively.

Figure 5 shows the detected cup boundary against 


\begin{tabular}{|l|cc|cc|cc|}
\hline & \multicolumn{2}{|c|}{ Expert-1 } & \multicolumn{2}{|c|}{ Expert-2 } & \multicolumn{2}{c|}{ Expert-3 } \\
\hline Cat/No. & $\mu$ & $\sigma$ & $\mu$ & $\sigma$ & $\mu$ & $\sigma$ \\
\hline $\mathbf{N} / 32$ & 0.56 & 0.79 & 0.23 & 0.19 & 0.18 & 0.28 \\
G/101 & 0.06 & 0.20 & 0.05 & 0.35 & 0.03 & 0.34 \\
\hline Total/133 & 0.09 & 0.80 & 0.09 & 0.33 & 0.01 & 0.34 \\
\hline
\end{tabular}

Table 1: Mean $\mu$ and $\sigma$ in CDR estimation.

three experts on a sample image (overlaid on segmented OD region). The proposed method successfully detects $r$-bends formed on both thick and thin vessel. The cup boundary at $r$-bends is closer to the experts' marked boundaries (fig. 5(a-2)), whereas in regions where they are absent the interpolated result is unable to match the boundary marked by the experts. We observed some challenging situations where our detected $r$-bends are not considered relevant by experts. For instance, in fig. 5(a-1) boundaries marked by experts are away from the detected $r$-bends though there was not $2 \mathrm{D}$ clue present to support their markings. These suggest the role of prior 3D knowledge of the cup being used by an expert to determine cup boundary.

Traditionally, the cup-to-disk ratio(CDR) in the vertical direction is used to quantify cup enlargement. This was computed from the obtained boundaries and compared against that from each of the experts. The average mean $\mu$ and standard deviation $\sigma$ of the CDR error for 32 normal and 101 glaucomatous images is shown in Table. 1. The method gives less estimation error against expert-3. The average error $\mu, \sigma$ over all three experts is $0.323,0.420$ for normal and $0.046,0.296$ glaucomatous images. These figures show that high accuracy of method in estimating CDR for glaucomatous images compared to normal image indicating high sensitivity in glaucoma detection.

The CDR measure is inadequate to assess local cup changes which is of clinical interest. We examined this by computing sector-wise cup segmentation accuracy.

An area (pixel) overlap-based method was used to compute the precision $(\mathrm{P})$-recall( $\mathrm{R})$ measures to assess the cup segmentation in each of the 4 sectors. Table 2 shows the sector-wise $\mathrm{P}$ and $\mathrm{R}$ measure computed over 133 images. These figures indicate that the method is consistent across four sectors and with an average precision $(0.79)$ and recall $(0.87)$.

In order to gain some insight into the reasons behind this performance we analysed the inter observer variance among the experts by taking E3 with 20 years experience, as our gold standard. The difference in cup radius between the gold standard and the experts were found for each point on the boundary (defined by the angle $\alpha$ ) for all 133 images. These are plotted in fig. 6 . It can be seen that on average the variance among the experts is $E 1=24.06$ and $E 2=23.91$. This indicates a fair degree of disagreement between experts and

\begin{tabular}{|c|c|c|c|c|c|c|c|c|}
\hline & \multicolumn{2}{|c|}{ Sec-1 } & \multicolumn{2}{c|}{ Sec-2 } & \multicolumn{2}{c|}{ Sec-3 } & \multicolumn{2}{|c|}{ Sec-4 } \\
\hline & $\mathrm{P}$ & $\mathrm{R}$ & $\mathrm{P}$ & $\mathrm{R}$ & $\mathrm{P}$ & $\mathrm{R}$ & $\mathrm{P}$ & $\mathrm{R}$ \\
\hline E-1 & 0.81 & 0.81 & 0.74 & 0.87 & 0.77 & 0.89 & 0.82 & 0.84 \\
\hline E-2 & 0.87 & 0.81 & 0.82 & 0.83 & 0.82 & 0.85 & 0.85 & 0.85 \\
\hline E-3 & 0.75 & 0.89 & 0.72 & 0.89 & 0.69 & 0.94 & 0.72 & 0.92 \\
\hline Avg & 0.81 & 0.84 & 0.76 & 0.86 & 0.76 & 0.89 & 0.80 & 0.87 \\
\hline
\end{tabular}

Table 2: Sector-wise precision and recall measures for cup segmentation.

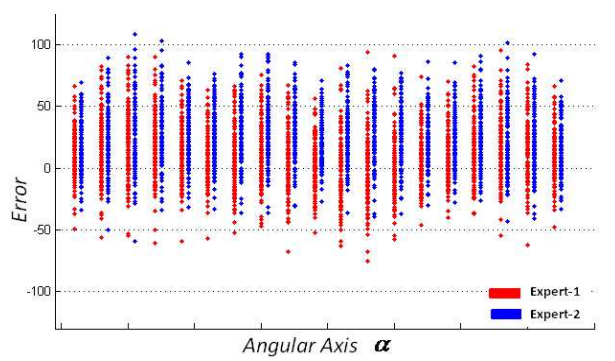

Fig. 6: Angular cup radius assessment against expert-3. attests to the complexity of the problem.

\section{Discussion and Conclusion}

In this paper, we presented a novel cup boundary detection method using $r$-bends information. Trench based vessel modeling and ROS-based bend detection that have been employed result in robustness to varying thickness of the vessels. Final cup boundary is obtained using applying local spline interpolation on the detected r-bends. Assessment results show that our method matches quite well with the most experienced expert's assessment. At a local level, the segmentation $\mathrm{P} / \mathrm{R}$ figures are $0.79 / 0.87$. It is observed that in the regions with no certain 2D clues, there is less consensus on the cup boundary between our method and experts and also within experts. This signals the ambiguity in $2 \mathrm{D}$ information and the importance of $3 \mathrm{D}$ information in cup segmentation which will be investigated in our future work.

\section{References}

[1] T. Chan and L. Vese. Active contours without edges. IEEE Trans. Image Processing, 10(2):266-277, 2001.

[2] S. Garg, J. Sivaswamy, and S. Chandra. Unsupervised curvature-based retinal vessel segmentation. Proc. ISBI, pages 344-347, 2007.

[3] J. Liu, D. Wong, J. Lim, H. Li, N. Tan, and T. Wong. Argali- an automatic cup-to-disc ratio measurement system for glaucoma detection and analysis framework. Proc. SPIE, Medical Imaging, pages 72603K-8, 2009.

[4] D. Wong, J. Liu, J. H. Lim, H. Li, X. Jia, F. Yin, and T. Wong. Automated detection of kinks from blood vessels for optic cup segmentation in retinal images. Proc. SPIE, Medical Imaging, page 72601J, 2009.

[5] J. Xu, O. Chutatape, E. Sung, C. Zheng, and P. Chew. Optic disk feature extraction via modified deformable model technique for glaucoma analysis. Pattern Recognition, 40(7):2063-2076, 2007. 